\title{
Sur le rôle des publications en mathématiques
}

Pierre Bérard

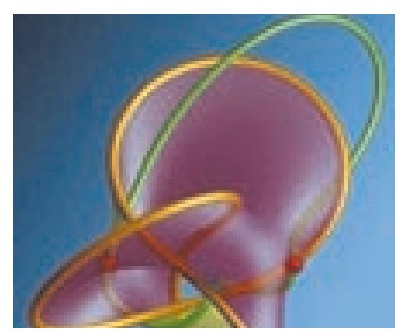

Université Joseph Fourier, Institut Fourier-

Mathématiques UJF-CNRS, BP 74, 38420 Saint

Martin d'Hères, France.

Pierre.Berard@ujf-grenoble.fr

http://www-fourier.

ujf-grenoble.fr/ pberard/

La littérature savante joue un rôle important dans toutes les disciplines scientifiques. En mathématiques, ce rôle est crucial. L'objet de cet article est d'expliquer pourquoi il en est ainsi, de préciser les besoins des mathématiciens en la matière et de présenter quelques actions menées par la communauté mathématique, en particulier en France.

\section{Le corpus mathématique}

Si les mathématiciens publient relativement peu, en revanche, ils lisent beaucoup. L'ensemble des publications mathématiques, en tant que corpus scientifique, forme un vaste réseau que nous désignerons ici par corpus mathématique. II constitue l'environnement dans et sur lequel les mathématiciens travaillent, dans lequel ils trouvent leur inspiration, les exemples qui forgent leur intuition, les outils pour faire aboutir leurs idées.

Ce corpus, les mathématiciens l'appréhendent et se l'approprient en l'analysant solitairement ou à plusieurs dans des groupes de travail ou des séminaires hebdomadaires, en échangeant avec leurs collègues à l'occasion d'ateliers, de congrès ou de séjours de recherche, en diffusant leurs travaux sous forme de pré-publications (c'est-à-dire d'articles non encore soumis à/acceptés par une revue), aujourd'hui par le biais d'entrepôts de données comme arXiv (http:// arxiv.org/) ou HAL (http://hal.archives-ouvertes.fr/), et, in fine, dans des revues à comité de lecture.

\section{Quelques chiffres en vrac}

Une quinzaine de revues contenaient des articles de mathématiques en 1700 ; elles étaient 200 à la fin du XVIII ${ }^{e}$ siècle. La première revue consacrée aux mathématiques a été créée en 1810 ; la revue mathématique vivante la plus ancienne remonte à 1826 . II faut souligner ici la féconde collaboration entre éditeurs commerciaux, sociétés savantes et institutions publiques dans le développement de ces revues. II se publiait moins de 1000 articles mathématiques par an dans la seconde moitié du XIXe siècle. Aujourd'hui, les bases de données mathématiques publient environ 75000 notices chaque année et elles dépouillent de manière exhaustive près de 600 revues mathématiques et, partiellement, des milliers de documents (au total plus de 1500 revues, des ouvrages, des actes de congrès, etc.).

Le Tableau / indique le nombre (chiffres arrondis) d'articles publiés dans des revues mathématiques à comité de lecture, et recensés dans les bases de données mathématiques $[1,2]$.

\begin{tabular}{|cc|}
\hline Période & Nombre d'articles \\
\hline $1860-1946$ & 250000 \\
\hline $1947-1956$ & 63000 \\
\hline $1957-1966$ & 120000 \\
\hline $1967-1976$ & 243000 \\
\hline $1977-1986$ & 335000 \\
\hline $1987-1996$ & 440000 \\
\hline $1997-2006$ & 614000 \\
\hline
\end{tabular}

Tableau I. Nombre (chiffres arrondis) d'articles publiés dans des revues mathématiques à comité de lecture. 
Sur les périodes 1987-1996 et 1997-2006, la part des articles dont au moins un auteur travaille en France, est respectivement de 4,65 et $5,90 \%$. En se limitant aux articles publiés dans les cinq meilleures revues, les parts correspondantes sont respectivement de 14,1 et $16,8 \%$. Ces derniers chiffres varient peu si l'on tient compte également du nombre de pages et si l'on partage les articles entre les différents pays de leurs co-auteurs $[1,3]$.

\section{Échelles de temps en mathématiques}

Les échelles de temps sont particulières en mathématiques. Le délai de publication d'un article dans une revue à comité de lecture est de l'ordre de deux ans, la demi-vie d'un article de dix à vingt ans, plus pour certains articles particulièrement importants. Plus précisément, des statistiques portant sur un large ensemble d'articles et comparant l'année de publication d'un article à celle des items cités, montrent qu'en moyenne $50 \%$ des références renvoient à des publications ayant moins de dix ans d'âge, et plus de $25 \%$ à des publications ayant au moins vingt ans d'âge. De fait, des idées ou des techniques mathématiques vieilles de plusieurs décennies, voire de cent ans d'âge, sont pertinentes, parfois cruciales, pour résoudre les problèmes contemporains en mathématiques ou dans leurs applications. Pour ces mêmes raisons, les traités et ouvrages de synthèse sont particulièrement importants en mathématiques, mais ils ne peuvent pas toujours remplacer la lecture de la source originale d'un résultat.

Du fait de ces échelles de temps particulières, l'indice de citation de I'ISI, basé sur une période de deux ans, est inadapté aux mathématiques. La base de données «Mathematical reviews » a introduit un indice plus pertinent, le «Mathematical citation quotient», basé sur une période de cinq ans (voir l'aide en ligne sur [1]). Les mathématiciens préfèrent en général fonder leurs évaluations sur la qualité des revues et, autant que possible, sur la lecture des travaux eux-mêmes. Voir [4] pour une réflexion sur les enjeux de la bibliométrie en mathématiques.

Enfin, il faut noter que les grandes avancées mathématiques sont souvent dues à la rencontre de sous-champs a priori bien distincts (algèbre et topologie par exemple). Cela est facilité par le fait que la majorité des revues mathématiques sont généralistes, ouvertes à toutes les sous-spécialités ou au moins à des sous-ensembles très larges.

Les mathématiques jouent, depuis le XVII e siècle, un rôle important dans les sciences, y compris plus récemment dans les aspects quantitatifs des sciences du vivant, en apportant des outils pour organiser, analyser, calculer et prédire. Le rôle des mathématiques dans le développement technologique s'amplifie chaque jour. À ce titre, le corpus mathématique est important non seulement pour la discipline ellemême, mais également pour les autres sciences et pour la technologie, et ce de manière asynchrone.

Un trait caractéristique des mathématiques parmi les sciences est, sans aucun doute, leur dépendance vis à vis du corpus mathématique. Les mathématiques ont prospéré depuis plus de 2500 ans parce que des informations clés ont été transmises de génération en génération par le biais du corpus mathématique. Ce corpus doit donc être très soigneusement validé, rangé, indexé, conservé, et rester accessible sur le très long terme.

\section{Quels enjeux pour le corpus mathématique?}

Les mathématiciens ont besoin de sources fiables

À partir du XVIII e siècle, les revues ont joué un rôle primordial comme moyen de validation et de conservation (grâce aux bibliothèques) d'éléments mathématiques clés. La robustesse du système de validation par les pairs a été garantie par la diversité de revues indépendantes, renommées et souvent issues d'une longue tradition.

La communication scientifique est devenue plus rapide et plus facile avec l'échange de fichiers numériques, et en particulier avec l'apparition de serveurs de documents électroniques. Cela se traduit également par la co-existence de versions successives d'un même document. Dans ce nouvel environnement, la validation par les pairs et la publication tendent à se déconnecter. Une préoccupation essentielle pour l'avenir des mathématiques est de préserver des sources fiables. Il est nécessaire en effet que les écrits mathématiques, validés par un comité de lecture, soient signalés, archivés et accessibles de manière pérenne.

\section{Les mathématiciens ont besoin}

\section{de l'ensemble du corpus mathématique}

L'utilisation, fréquente en mathématiques, de résultats anciens, rend cruciales les questions de préservation et d'archivage. Les bibliothèques ont, jusqu'à ce jour, joué un rôle central à cet égard. L'apparition depuis quelques années des licences d'accès pose de nouvelles questions, de nature plus politique.

- Qui sera responsable à l'avenir de l'archivage (accès et préservation) du patrimoine mathématique?

- La production mathématique continuera-t-elle de faire partie de notre héritage commun ou bien serat-elle graduellement confisquée au profit d'intérêts particuliers?

Compte tenu de l'importance de la littérature mathématique pour la discipline elle-même, comme pour l'avancement de la science et de la technologie, il nous paraît indispensable pour le corpus mathématique que les missions de préservation, d'archivage et de fourniture d'accès à long terme soient partagées, dans un effort international, entre les communautés et les institutions académiques (en utilisant des standards ouverts). Ces missions ne peuvent pas être confiées à la seule responsabilité de groupes commerciaux dont elles ne sont pas le souci majeur. L'Union mathématique internationale a approuvé une recommandation en ce sens, ([5], recommandation 14 ).

S'il paraît difficile, voire impossible, de prédire le futur de la publication scientifique, il reste que la recherche 
se fait aujourd'hui à travers des moyens électroniques et que la possibilité d'accès d'un seul clic à des travaux de référence est un enjeu majeur.

La communauté mathématique internationale rêve de transformer le corpus mathématique en une immense Bibliothèque mathématique numérique (voir la déclaration [6]). Génération charnière entre l'ère du papier et l'ère électronique, nous avons le devoir de transmettre notre héritage fondamental aux générations futures et de réaliser la conversion de la littérature imprimée vers un format numérique aussi riche que possible, pour permettre les adaptations futures.

La Bibliothèque mathématique numérique devrait constituer une collection complète contenant à la fois des documents rétro numérisés (numérisés à partir de versions papier) et des documents nativement sous forme numérique, et répondre aux trois fonctions fondamentales d'une bibliothèque : l'archivage pour l'accès et la préservation pérennes, l'acquisition. L’idée générale est que les données brutes (fichiers numériques) soient d'accès libre sur la Toile, permettant ainsi à des sociétés savantes, des institutions ou des éditeurs de fournir des services à valeur ajoutée et des améliorations (liens croisés, commentaires), éventuellement avec un accès payant (voir [7] pour un scénario possible).

L'Union mathématique internationale a approuvé une recommandation pour un accès non restreint à certaines données telles que sommaires, résumés, mots-clés (et, dans la mesure du possible, bibliographies), et une recommandation pour un accès libre à terme (accès libre aux textes intégraux après un certain laps de temps) (voir [5], recommandations 11 et 12).

Ce rêve n'est malheureusement pas encore une réalité, malgré plusieurs initiatives importantes (y compris en France) et une certaine coopération internationale entre les acteurs depuis 2002. Ce qui fait défaut aujourd'hui c'est une réelle coordination des efforts, sous-tendue par un financement important, en particulier au niveau européen. (voir [8] pour un état des lieux sans concession).

En donnant un large accès au corpus mathématique, notre héritage commun, la Bibliothèque mathématique numérique pourrait avoir un impact important sur la manière dont les mathématiques seront faites et utilisées au XXI siècle, un impact qui pourrait être aussi important que celui de l'émergence des revues il y a trois siècles, voire celui de l'imprimerie.

Les mathématiciens ont besoin d'organisation et de liens croisés Les mathématiciens (et d'autres bien sûr) ont reconnu très tôt le besoin de disposer d'outils pour organiser un corpus en expansion constante et s'y frayer un chemin. Cela a conduit à l'émergence de classifications thématiques et de bases de données ou, plus exactement dans le domaine des mathématiques, de bases de recensions (les articles ne sont pas seulement signalés, ils sont également commentés par des experts et des références croisées sont introduites).

La première base de données mathématiques (Jahrbuch über die Fortschritte der Mathematik) a été créée en 1868. Disparue en 1942, elle est aujourd'hui numérisée et intégrée au Zentralblatt MATH [2], l'une des deux bases de données, avec les Mathematical reviews [1], qui couvrent la littérature mathématique de manière exhaustive en utilisant la même classification par sujets. Ces bases comportent des liens internes et des liens vers les articles (plein texte). En incluant les fonds rétro numérisés et les bibliographies des articles dans les recensions, elles constitueront à terme un pivot pour explorer les interconnexions à l'intérieur du corpus mathématique, en particulier dans le cadre de la Bibliothèque mathématique numérique (voir [9] pour plus de détails). Le protocole OAI-PMH (Open archives initiative's protocol for metadata harvesting) apporte également une réponse à ce besoin d'organisation en permettant le développement d'interfaces de recherche fédératives, comme le projet «Mini DML» (http:// minidml.mathdoc.fr/).

\section{Que fait la communauté mathématique?}

Les mathématiciens se sont toujours souciés des questions de publication. Nombre d'institutions académiques et de sociétés savantes ont une longue tradition de publication de revues internationales de premier plan et d'ouvrages de référence. Pour répondre aux enjeux de l'ère numérique, l'Union mathématique internationale (IMU) et la Société mathématique européenne (EMS) ont mis en place des comités sur la communication et la publication électroniques (http://www.ceic.math.ca/ et http://www.emis.de/committees.html).

La France participe de cette longue tradition. Une vingtaine de revues de mathématiques sont aujourd'hui publiées sous l'égide d'une institution ou, plus généralement, de la communauté mathématique françaises. Certaines sont historiques. Deux d'entre elles, les Publications mathématiques de l'Institut des hautes études scientifiques et les Annales scientifiques de l'École normale supérieure, figurent parmi les revues mathématiques les plus citées au monde.

Plus récemment, et pour faire face aux enjeux actuels de l'information scientifique et technique en mathématiques, deux structures nationales ont été créées, le Réseau national des bibliothèques de mathématiques (Groupement de service du CNRS, http://www.rnbm. org/) et la Cellule MathDoc, un Institut pour l'information et la communication scientifiques (Unité mixte de service CNRS - Université Joseph Fourier, Grenoble). Ces structures travaillent en lien étroit avec les directions scientifiques concernées au Ministère chargé de la recherche et au CNRS.

Par l'intermédiaire de la Cellule MathDoc, la France prend une part active à la transformation de la base de données Zentralblatt MATH en un grand instrument européen au service de la communauté mathématique 
internationale, sous l'égide de la Société mathématique européenne. La Cellule MathDoc pilote deux programmes nationaux, NUMDAM et CEDRAM, dont l'objectif commun est de soutenir les revues mathématiques françaises. Ils sont détaillés ci-dessous.

\section{Enjeux et objectifs}

Les enjeux qui ont motivé la mise en place du programme NUMDAM, puis de CEDRAM, sont résumés par les mots-clés visibilité, accès et archivage à long terme. La conception de ces programmes a été influencée par JSTOR (Journal storage) ${ }^{1}$ et DIEPER (DIgitised European PERiodicals $)^{2}$, et par des discussions en France et au niveau international (Union mathématique internationale et Société mathématique européenne).

Deux objectifs majeurs ont été pris en compte: (1) construire une archive numérique de l'héritage mathématique «français » (au sens des supports, pas de la nationalité des auteurs), qui serait ensuite entretenue par une institution publique; et (2) renforcer la présence des revues mathématiques françaises sur la Toile, de telle sorte qu'elles soient compétitives en termes de fonctionnalités (l'étant déjà sur le plan scientifique). Voir [8] pour une description plus détaillée.

\section{NUMDAM}

Créé en 2000, l'objectif du programme de NUMérisation de Documents Anciens Mathématiques (http://www.numdam.org) est de numériser l'ensemble de la littérature mathématique publiée en France sous forme imprimée et de participer au projet international de Bibliothèque mathématique numérique.

Le programme a débuté par une longue période (deux ans) de définition du cahier des charges et des procédures. Les deux premières phases, terminées fin 2007, ont permis la numérisation de près de 600000 pages, soit 27000 articles (25 collections différentes, dont 3 européennes non françaises et 19 collections vivantes).

Les choix techniques ou politiques tiennent compte des besoins des utilisateurs.

- Pages scannées à 600 ppp pour un bon rendu des formules complexes et pour permettre des traitements ultérieurs en fonction des évolutions technologiques (reconnaissance de structure, de formules).

- Reconnaissance optique de caractères pour permettre les recherches plein texte.

${ }^{1}$ À l'origine, JSTOR émane de la Fondation Andrew W. Mellon (États-Unis) et cherche à déterminer si la numérisation permet de réduire les coûts de stockage des collections de périodiques et d'améliorer en même temps l'accès à celles-ci. En cinq ans, cette organisation à but non lucratif a engrangé dans ses bases le texte de 120 titres de périodiques libres de droits en remontant loin dans le XIXe siècle, ce qui représente cinq millions de pages dans quinze disciplines différentes. Sept cents institutions académiques participent à ce jour à ce travail collectif, dont 550 aux États-Unis. JSTOR est l'abréviation de Journal STORage. Ce nom indique à première vue qu'il s'agit d'un entrepôt de périodiques. Mais il y a plus, storage traduit aussi l'idée de rangement et de conservation. JSTOR a pour but de trouver une solution électronique aux problèmes d'espace vécus par les bibliothèques qui soit à la fois économique et qui permette d'améliorer l'accès et la conservation des publications savantes. II ne s'agit pas d'abonnements courants, mais bien de collections rétrospectives. Les plus anciens numéros remontent à 1831, les plus récents, à 2006. En 2007, JSTOR archive et diffuse plus de 700 périodiques, ce qui représente plus de $23,706,100$ pages-images [source http://www.bibl.ulaval.ca].

${ }^{2}$ Voir le site SUB Goettingen : http://dz-srvl.sub.uni-goettingen.de/GDZ/dieper/ et également le site d'EROMM: http://www.eromm.org/ car le contenu de la base de données de Dieper a été versé dans celle d'EROMM.
- Segmentation précise pour une bonne granularité (niveau des articles).

- Saisie et balisage des bibliographies qui font partie intégrante de la base de données.

- Liens vers les bases de données mathématiques chaque fois que cela est possible (au niveau des articles numérisés eux-mêmes et au niveau des références bibliographiques qu'ils contiennent).

- Les métadonnées (données de catalogage, résumés, bibliographies, texte traité par reconnaissance optique caché) des documents numérisés sont librement accessibles sur la Toile.

- Le texte intégral des articles (mode image) est disponible librement sur la Toile après un certain laps de temps (créneau mobile) afin d'assurer la stabilité économique des revues.

Le créneau mobile recommandé est de 5 ans (les articles publiés l'année $N$ sont accessibles librement à partir de l'année $N+5$ ), ce qui est compatible avec la demi-vie moyenne d'un article de mathématiques (10 à 20 ans).

Un aspect important est le caractère vivant de l'archive. Les collections numérisées à partir du papier sont aujourd'hui complétées avec la production nativement numérique des revues (avec le même créneau mobile). Les conditions de mise à disposition sont déterminées, au cas par cas, après négociation avec les institutions et éditeurs concernés. Les questions de droit d'auteur sont également traitées.

L'objectif du programme NUMDAM aujourd'hui est de développer des collections numériques de référence (y compris des archives, des thèses historiques,...) et de nouvelles fonctionnalités.

Parmi les autres fonds mathématiques numériques qui sont aujourd'hui disponibles en France, le plus important est celui de la Bibliothèque nationale de France (http://gallica.bnf.fr/ : Comptes rendus de l'Académie des sciences jusqu'en 1964, ouvrages, oeuvres complètes, etc., voir également le portail spécifique http:// math-doc.ujf-grenoble.fr/GALLICA/).

On ne peut que regretter que la plus ancienne revue française vivante de mathématiques (le Journal de mathématiques pures et appliquées, créé en 1836 par Joseph Liouville) ne figure que partiellement dans l'archive (à travers BnF - Gallica), faute d'accord avec l'éditeur actuel, Elsevier.

\section{CEDRAM}

Le Centre de Diffusion des Revues Académiques de Mathématiques (http://www.cedram.org) est un service de la Cellule MathDoc. II répond aux objectifs de visibi- 
lité, de présence sur la Toile et dans les bases de données mathématiques, et de préservation de la production courante des revues. II s'agit d'un effort concerté des revues participantes elles-mêmes, de la Cellule MathDoc et d'un comité de pilotage auquel participent les deux sociétés françaises de mathématiques.

CEDRAM offre aux revues un ensemble d'outils de production (éditions électronique et imprimée), adaptables aux caractéristiques de chaque revue, et une plate-forme d'hébergement. La face visible est un portail (liens vers les sites des revues, feuilletage des collections et moteur de recherche commun). La plate-forme offre les mêmes fonctionnalités que NUMDAM (en particulier, base de données enrichie, bibliographies des articles comportant des liens vers les bases de données mathématiques), ainsi qu'un contrôle d'accès aux textes intégraux. L'objectif de préservation est décliné en deux stratégies, l'une de préservation à long terme des fichiers destinés aux utilisateurs (conversion des méta données au format NUMDAM, avec le même créneau mobile) et l'autre de préservation des données de production pour que les sources restent au plus près du contenu mathématique publié.

Chaque journal reste indépendant sur le plan scientifique et sur le plan financier, la possibilité d'offrir des abonnements groupés est envisagée.

\section{Conclusion}

La prospérité des mathématiques, comme discipline scientifique à part entière, et comme discipline au service des autres sciences et des technologies, dépendra de la manière dont nous continuerons à enrichir, à préserver et à archiver le corpus mathématique, notre héritage commun. La prospérité des mathématiques dépendra aussi du fait que ce corpus continuera de faire partie intégrante du patrimoine de l'humanité et qu'il ne soit pas graduellement confisqué au bénéfice d'intérêts particuliers.

Nous devons être particulièrement attentifs à ce que les choix et les décisions d'aujourd'hui ne mettent pas en péril demain notre riche héritage. $\diamond$

\section{About the role of mathematics publications}

\section{RÉFÉRENCES}

1. Mathematical reviews. http://www.ams.org/mathscinet/

2. Zentralblatt MATH. http://www.zentralblatt-math.org/zmath/en/ advanced/

3. Schlenker Jean-Marc. Communication privée, 2008.

4. Schlenker Jean-Marc. Les enjeux de la bibliométrie pour les mathématiques, Société mathématique de France. Gazette des Mathématiciens 2008 ; 115 : 73-9.

http://smf.emath.fr/Publications/Gazette/2008/115/smf_gazette_ 115_73-79.pdf (dernière visite 22 janvier 2008).

5. CEIC. Best current practices: recommendations on electronic information and communication (2002), endorsed by the executive committee of the international mathematical union on april 13, 2002. CEIC, Committee on Electronic Information and Communication.

http://www.ceic.math.ca/Publications/Recommendations/BPs.pdf (dernière visite 22-01-2008)

6. CદIC. Digital mathematics library: a vision for the future, 2005. http://www.ceic.math.ca/Publications/dml_vision.pdf (dernière visite 22 janvier 2008).

7. Ewing John. Twenty centuries of mathematics: digitizing and disseminating the past mathematical literature. Notices of the American Mathematical Society $2002 ; 49: 771-7$.

http://www.ams.org/notices/200207/fea-ewing.pdf (dernière visite 22 janvier 2008).

8. Bouche Thierry. Towards a digital mathematics library. In : Borwein Jon, Rocha Eugénio, Rodrigues José, eds. Communicating mathematics in the digital era. AK Peters Ltd, 2008 : 43-67. http://www-fourier.ujf-grenoble.fr/ bouche/DML/bouche-dml.pdf (dernière visite 23-01-2008).

9. Bérard Pierre. Documentation issues for mathematics in the digital age. INSPEL 2002 ; 36 : 147-61. http://www.ifla.org/VII/d2/inspel/02-3bepi. pdf (dernière visite $1^{\text {er }}$ janvier 2008).

\section{REMERCIEMENTS}

L'auteur remercie Thierry Bouche, Laurent Guillopé et Jean-Marc Schlenker pour leurs commentaires sur une version préliminaire de cet article.

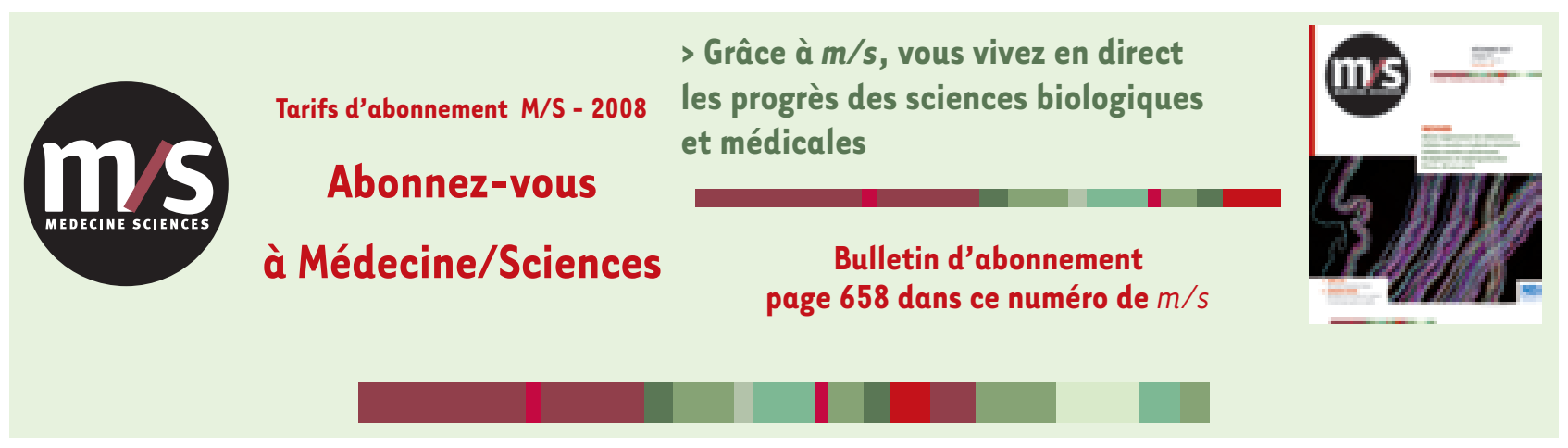

TIRÉS À PART

P. Bérard 


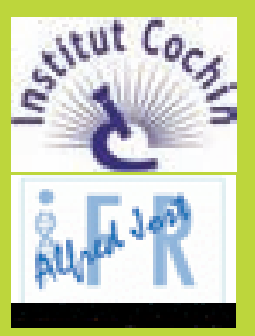

\section{Journéc Jean-CLaude Dreyfus \\ Molecular and Cellular Basis of Cell Motility \\ Bases Moléculaires et Cellulaires de la Motilité}

VENDREDI 26 SEPTEMBRE 2008

24, rue du Faubourg St Jacques - 75014 PARIS, France

(Métro : St Jacques - RER B : Port-Royal - Bus : 91, 83)

\section{8 h45 - Opening ceremony by Axel Kahn, President of the University Paris Descartes Ouverture par Axel Kahn, Président de l'Université Paris Descartes}

Molecular basis of cell motility

chairpersons: Jérôme Delon et Florence Niede

Marie-France Carlier, Laboratoire d'Enzymologie et Biochimie Structurales, Gif-sur-Yvette, France

"Mechanism of actin-based motility: from molecules to movement"

Cécile Sykes, Institut Curie, Paris, France

"A step towards artificial cell movement"

François Guillemot, National Institute for Medical Research, London, UK

"Transcriptional control of neuronal migration in the developing forebrain"

\section{Guidance cues, signalling and migration (I)}

Ronen Alon, Weizmann Institute of Science, Rehovot, Israel

"A new dimension in chemokine triggered lymphocyte migration across endothelial barriers"

Matthew Krummel, University of California, San Francisco, USA

"Cytoskeletal arrays underlying T cell motility and their reappropriation during synapse establishment"

Robert Insall, Beatson Institute for Cancer Research, Glasgow, UK

"Pseudopods, SCAR/WAVE and the mechanisms that drive chemotaxis"

\section{Guidance cues, signalling and migration (II)}

Pascal Silberzan, Institut Curie, Paris, France

"Collective migration of epithelial cells"

Sophie Chauvet, Institut de biologie du développement de Marseille Luminy, Marseille, France

"Semaphorin $3 \varepsilon$ : function and signalling in neural development"

Erez Raz, Institute of Cell Biology, Münster, Germany

"Molecular and cellular mechanisms controlling germ-cell migration in Zebrafish"

Michael Sixt, Max Planck, Institute of Biochemistry, Martinsried, Germany

"Chemokine gradient sensing within lymphatic organs"

\section{Misregulation in migratory processes}

chairpersons: Emmanuel Donnadieu et François Guillemot

Pierre Gressens, Hôpital Robert Debré, Paris, France

"Disorders of neuronal migration"

Alexander Flügel, Max Planck Institute of Neurobiology, Martinsried, Germany

"How brain-autoaggressive T cells enter their target organ"

Cédric Gaggioli, Cancer Research UK, London, UK

"Collective invasion of carcinoma cells is led by the fibroblasts"

APEMM CONGRES, Institut Cochin, Michelle Liu, 22 rue Méchain - 75014 PARIS - Tél 0140516463 - email : michelle.liu@inserm.fr

Registrations before 5 th September 2008/Inscriptions avant le 5 septembre : $55 €$

Payment/Paiement : international payment, bank transfer (contact us) par chèque au nom de APEMM Gestion (Association loi 1901) ou bon de commande administratif. 